\title{
Métodos anticonceptivos elegidos por mujeres después de aborto en un hospital público de Honduras
}

Contraceptive methods chosen by women after abortion in a public hospital in Honduras

\author{
Rigoberto Castro (1) https://orcid.org/0000-0001-7445-306X, Jamilette Paz 난 https://orcid.org/0000-0002-0804-4775.
}

Secretaría de Salud, Hospital Dr. Roberto Suazo Córdova, La Paz, Honduras.

RESUMEN. Antecedentes: La tasa de embarazos no planeados en Latinoamérica está entre las más altas del mundo. Las adolescentes están entre los grupos más vulnerables para embarazos no planeados con porcentajes de $32 \%$ a $45 \%$. Objetivo: Describir los factores asociados a la elección de un anticonceptivo por las mujeres después de un aborto, en el Hospital Dr. Roberto Suazo Córdova, La Paz, Honduras, en el periodo de julio 2017 a junio 2019. Método: Estudio retrospectivo descriptivo. Los datos fueron obtenidos usando el Sistema Informático Perinatal Aborto (SIP Aborto) en la versión SIP 4.16. Resultados: El 50.0\% (67/134) de las adolescentes y $50.1 \%$ (281/550) de las mayores de 19 años eligieron un anticonceptivo postaborto. El inicio del anticonceptivo se relacionó con antecedente de aborto $(p=0.006)$. Uso previo de anticonceptivos $(p=0.007)$. Los anticonceptivos más elegidos fueron: Acetato de medroxiprogesterona, anticonceptivos orales combinados y condón. La elección del anticonceptivo se relacionó con evacuación instrumental del aborto $(p=0.022)$ en adolescentes y $(p=0.000)$ en mayores de 19 años. Se eligieron menos los métodos reversibles de acción prolongada. Discusión: La elección anticonceptiva postaborto fue baja. El inicio de anticonceptivos se relacionó con antecedente de aborto, uso previo de anticonceptivos y evacuación instrumental. Los anticonceptivos más elegidos fueron los temporales de acción corta y los menos elegidos, los temporales de acción prolongada. Se debe fortalecer el acceso a información, mejorar la consejería, garantizar acceso y disponibilidad de anticonceptivos sobre todo de acción prolongada para asegurar el apego al método anticonceptivo.

Palabras claves: Aborto, Adolescentes, Anticonceptivos, Consejería.

\section{INTRODUCCIÓN}

En América Latina y el Caribe (LAC) existen grandes disparidades en desarrollo socioeconómico, acceso a los servicios de salud incluido atención en Salud Sexual y Reproductiva y Derechos (SSRD). El garantizar el acceso universal a la SSRD es una estrategia fundamental para mejorar la salud materna y reducir la cantidad de embarazos no planeados, morbimortalidad materna e infantil y la cantidad de abortos inseguros. ${ }^{1}$

La tasa de embarazos no planeados en LAC todavía se encuentra entre las más altas del mundo. Se considera que más del $60 \%$ de los embarazos en la región son no planeados relacionado esencialmente con falta de uso de Métodos Anticonceptivos (MAC), falla del MAC y uso incorrecto del mismo. ${ }^{1}$ También, debe tenerse en consideración que el acceso a una Interrupción Voluntaria del Embarazo (IVE) es restringido o prohibido en muchos países en LAC. Entre estos países podemos mencionar Honduras, ya que la legislación penal del país prohíbe la interrupción de un embarazo bajo cualquier circunstancia; a pesar de ello, se estima que entre el $40 \%$ al $42 \%$ de los embarazos no planeados terminan en IVE. ${ }^{1}$ Esto conlleva a que las mujeres sean más propensas a un aborto inseguro con las consecuentes complicaciones y riesgo de morbi-mortalidad.

Se ha estimado que entre el $8 \%$ al $18 \%$ de las muertes maternas a nivel mundial son debido a abortos inseguros. ${ }^{2}$ Esta situación se ha reconocido como una de las principales preocupaciones de la salud pública a nivel mundial sobre todo porque cerca de un millón de mujeres son hospitalizadas en LAC anualmente para ser tratadas por complicaciones derivadas de abortos inseguros siendo causa de muertes maternas en esta región. ${ }^{3}$
Recibido: 15-07-2020 Aceptado: 01-09-2021 Primera vez publicado en línea: 05-10-2021 Dirigir correspondencia a: Dr. Rigoberto Castro Correo electrónico: rcastrob561@hotmail.com

DECLARACIÓN DE RELACIONES Y ACTIVIDADES FINANCIERAS Y NO FINANCIERAS: Este artículo se realizó con el apoyo financiero de la Organización Mundial de la Salud/Organización Panamericana de la Salud (OMS/OPS) y apoyo técnico del Centro Latinoamericano de Perinatología, Salud de la Mujer y Reproductiva (CLAP/SMR).
Forma de citar: Castro R, Paz J. Métodos anticonceptivos elegidos por mujeres después de aborto en un hospital público de Honduras. Rev Méd Hondur. 2021; 89 (2): 96-102. DOI: https://doi.org/10.5377/rmh.v89i2.12526

○ 2021 Autor(es). Artículo de acceso abierto bajo la licencia https://creativecommons.org/ licenses/by/4.0/deed.es

DECLARACION DE CONFLICTOS DE INTERÉS: Ninguno 
En un estudio realizado en Honduras entre los años 2010 y 2011 en hospitales públicos de pacientes atendidas por aborto, se encontró que el $20.8 \%$ eran adolescentes. ${ }^{3}$

El $68.6 \%$ de todas las mujeres en situación de aborto iniciaron un $\mathrm{MAC},{ }^{3}$ si bien no fue indicada la proporción de adolescentes que inició un MAC, uno de los grupos más vulnerables para embarazos no planeados han sido las adolescentes, estimándose que la tasa se ha mantenido alta o se ha incrementado. ${ }^{1}$

La evidencia indica que entre el $32 \%$ al $45 \%$ de los embarazos en adolescentes son no planeados. ${ }^{1}$ Estudios de poblaciones han mostrado que el primer embarazo temprano y no deseado son comunes y las mujeres jóvenes han reconocido el uso de la anticoncepción como la clave para prevenir estos embarazos. ${ }^{4}$

Los embarazos repetidos en adolescentes aún son un fenómeno generalizado en países de bajo y mediano ingreso, siendo que algunos de los factores de riesgo reportados son antecedente de aborto espontáneo y falta de uso o uso inadecuado de MAC. Entre los factores protectores contra el embarazo repetido se ha encontrado un alto nivel de escolaridad y el apoyo emocional familiar. Estos hallazgos tienen grandes implicancias para la planificación de futuras intervenciones orientadas a prevenir embarazos repetidos en adolescentes. ${ }^{5}$ El conocimiento y valoración que las adolescentes tienen sobre el costo y beneficio de los MAC y el embarazo, la consejería y el acceso a los métodos anticonceptivos después de un aborto son factores importantes que influyen en los cuidados anticonceptivos postaborto. ${ }^{5-7}$

Hallazgos de investigaciones recientes recomiendan que las adolescentes inicien la anticoncepción inmediatamente después del aborto ya que la motivación para elegir un método más efectivo puede ser mayor en este momento, preferiblemente un Método Reversible de Acción Prolongada (LARC, por su sigla en inglés) ya que estos son seguros y los más efectivos. ${ }^{8}$ Las mujeres que deciden interrumpir voluntariamente un embarazo pueden estar más motivadas a elegir un LARC, en comparación con aquellas mujeres sin antecedentes de aborto. Por lo que fomentar el acceso a un MAC LARC postaborto inmediato es esencial para prevenir la repetición de embarazos no planeados. $^{9}$

El Hospital Dr. Roberto Suazo Córdova, es uno de los 5 hospitales que en representación de Honduras forma parte de la Red MUSA (Mujeres en Situación de Aborto), que ha sido estructurada por la OPS y coordinada por el CLAP y que se extiende por Latinoamérica y el Caribe. Tiene como parte de sus objetivos garantizar una vigilancia adecuada de las mujeres que han tenido un aborto y promover la investigación. ${ }^{10}$ El hospital cuenta con el SIP como herramienta para procesamiento de los registros médicos de las pacientes ingresadas con diagnóstico de parto o aborto para la creación de una base de datos; por medio del sistema SIP Aborto se procesa la información relacionada con la atención postaborto. Debido a la falta de información en nuestro país, el objetivo del estudio fue describir los factores asociados en la elección de un método anticonceptivo por las mujeres después de un aborto.

\section{MATERIALES Y MÉTODOS}

Se realizó un estudio retrospectivo descriptivo, en el que se consideraron todas las mujeres en situación de aborto que fueron atendidas en el periodo de estudio de julio 2017 a junio 2019 en el Hospital Dr. Roberto Suazo Córdova, La Paz, Honduras. Se incluyeron expedientes de mujeres que fueron admitidas por un aborto independientemente del procedimiento de evacuación, la decisión de iniciar un método anticonceptivo o cual fue el método elegido. Para propósitos del estudio no se excluyó ninguna mujer admitida por aborto.

El sitio del estudio fue un hospital público, que atiende pacientes de mediano y alto riesgo, de la Secretaría de Salud de Honduras con un área de influencia de aproximadamente 222,000 habitantes; se atiende aproximadamente 3,600 partos al año y cuenta con 20 camas en el área de obstetricia y una baja mortalidad materna.

Para este estudio se utilizaron fuentes secundarias de información tales como los registros médicos y la base de datos electrónica del Hospital Dr. Roberto Suazo Córdova. Estos datos fueron obtenidos usando el SIP Aborto en la versión SIP 4.16. Esta base de datos se obtuvo de la digitalización de los registros médicos por personal previamente capacitado en el llenado de la historia clínica electrónica del SIP 4.16. Posteriormente se realizó un control de calidad de la base de datos del SIP, eligiéndose $10 \%$ de los expedientes electrónicos y se comparó con los respectivos registros médicos físicos para analizar inconsistencias, observándose que no se presentó ninguna inconsistencia entre los expedientes electrónicos y los registros médicos físicos. Para el análisis estadístico se usó el programa Social Science Statistic Calculator vía web.

Todas las mujeres admitidas después de un aborto durante el periodo de estudio fueron divididas en dos grupos según la edad al momento del aborto en adolescentes (de 10 a 19 años) y adultas (mayores de 19 años). Posteriormente ambos grupos fueron estratificados de acuerdo al inicio de un método anticonceptivo, método elegido después del aborto y se analizaron según las variables nivel educativo, estado marital, antecedentes obstétricos, embarazo planeado, uso previo de métodos anticonceptivos, consejería en anticoncepción, tipo de evacuación uterina ya sea tratamiento médico o evacuación quirúrgica Aspiración Manual Endouterina (AMEU), Legrado Uterino Instrumental (LUI) como variables independientes, como variable dependiente el postaborto.

Se realizó un análisis univariado de las variables cualitativas estableciendo frecuencias absolutas y relativas y el análisis bivariado se realizó usando la prueba de chi cuadrado, considerando un valor $p<0.05$ como estadísticamente significativo. El estudio fue aprobado por el Comité de Ética del Hospital Dr. Roberto Suazo Córdova. (Número 31072019) 


\section{RESULTADOS}

Se analizaron 684 historias clínicas de mujeres que fueron admitidas por aborto espontáneo, el 19.6\% (134) pertenecía al grupo de 10 a 19 años y $80.4 \%$ (550) a mujeres mayores de 19 años. El $54.4 \%$ (372) tenía estudios de primaria y $34.2 \%$ (234) de secundaria. El 29.8\% (204) no tenía antecedentes de embarazos previos y un $18.3 \%$ (125) tenía el antecedente de un aborto anterior. El 50.4\% (345) de los embarazos fue planeado

Cuadro 1. Características sociodemográficas y obstétricas de las mujeres admitidas por aborto, Hospital Dr. Roberto Suazo Córdova, julio 2017 a junio 2019, $\mathrm{n}=684$.

\begin{tabular}{|c|c|c|}
\hline Edad (años) & $\mathrm{n}$ & $(\%)$ \\
\hline $10-14$ & 5 & $(0.7)$ \\
\hline $15-19$ & 129 & $(18.8)$ \\
\hline $20-24$ & 182 & $(26.6)$ \\
\hline $25-29$ & 157 & $(22.9)$ \\
\hline $30-34$ & 97 & $(14.2)$ \\
\hline$>35$ & 114 & (16.8) \\
\hline \multicolumn{3}{|l|}{ Nivel de escolaridad } \\
\hline Ninguno & 36 & $(5.3)$ \\
\hline Primaria & 372 & $(54.4)$ \\
\hline Secundaria & 234 & $(34.2)$ \\
\hline Universitaria & 42 & $(6.1)$ \\
\hline \multicolumn{3}{|l|}{ Estado marital } \\
\hline Sin Pareja & 77 & $(11.3)$ \\
\hline Con Pareja & 607 & $(88.7)$ \\
\hline \multicolumn{3}{|l|}{ Número de gestas } \\
\hline 0 & 204 & $(29.8)$ \\
\hline $1-3$ & 392 & $(57.3)$ \\
\hline$>3$ & 88 & $(12.9)$ \\
\hline Abortos Previos & 125 & $(18.3)$ \\
\hline \multicolumn{3}{|l|}{ Este embarazo fue planeado } \\
\hline $\mathrm{Si}$ & 345 & $(50.4)$ \\
\hline \multicolumn{3}{|c|}{ Uso previo de método anticonceptivo } \\
\hline Si usaba & 101 & $(14.8)$ \\
\hline \multicolumn{3}{|l|}{ Anticonceptivos } \\
\hline Acetato de Medroxiprogesterona & 135 & $(19.7)$ \\
\hline Condón & 86 & (12.6) \\
\hline Combinados orales & 77 & $(11.3)$ \\
\hline Dispositivo Intrauterino & 26 & $(3.8)$ \\
\hline Ligadura tubaria & 11 & $(1.6)$ \\
\hline Otro hormonal & 5 & $(0.7)$ \\
\hline Otros de barrera & 4 & $(0.6)$ \\
\hline Implantes & 2 & $(0.3)$ \\
\hline Abstinencia periódica & 2 & $(0.3)$ \\
\hline Sin Anticonceptivos & 336 & $(49.1)$ \\
\hline \multicolumn{3}{|l|}{ Recibió consejería anticonceptiva } \\
\hline $\mathrm{Si}$ & 681 & (99.6) \\
\hline
\end{tabular}

y el $14.8 \%$ (101) de las mujeres uso un método anticonceptivo previo al embarazo (Cuadro 1).

El 50.0\% (67/134) de las mujeres de 10-19 años tuvo un embarazo no planeado y $91.8 \%$ (123) no usó un MAC. El 99.2\% (133) recibió consejería en planificación familiar (Cuadro 2).

Según el método de evacuación uterina, en las adolescentes el $63.4 \%$ (85) el método de evacuación fue quirúrgico y dentro de este método de evacuación, el procedimiento quirúrgico más practicado fue el AMEU que se practicó en el 90.6\% (77) de los 85 procedimientos y el tratamiento médico se realizó en el $33.6 \%$ (45). En las mujeres mayores de 19 años el tratamiento operatorio se practicó en el $58.2 \%$ (320), la evacuación por AMEU fue la que más se realizó con $89.7 \%$ (287) de los 320 procedimientos. Mientras que el $40.0 \%$ (220) se aplicó tratamiento médico (Cuadro 2).

Cuadro 2. Características sociodemográficas y obstétricas según grupos de edad de mujeres admitidas por aborto, Hospital Dr. Roberto Suazo Córdova, julio 2017 a junio $2019, n=684$.

\begin{tabular}{|c|c|c|c|c|}
\hline \multirow[t]{2}{*}{ Características } & \multicolumn{2}{|c|}{$\begin{array}{c}\text { Adolescentes 10- } \\
19 \text { años }(n=134)\end{array}$} & \multicolumn{2}{|c|}{$\begin{array}{c}\text { Mujeres }>19 \text { años } \\
\quad(n=550)\end{array}$} \\
\hline & $\mathrm{n}$ & $(\%)$ & $\mathrm{n}$ & $(\%)$ \\
\hline \multicolumn{5}{|l|}{ Nivel de escolaridad } \\
\hline Ninguno & 3 & $(2.2)$ & 33 & $(6.0)$ \\
\hline Primaria & 71 & $(53.0)$ & 301 & $(54.7)$ \\
\hline Secundaria & 56 & $(41.8)$ & 178 & $(32.4)$ \\
\hline Universitaria & 4 & $(3.0)$ & 38 & $(6.9)$ \\
\hline \multicolumn{5}{|l|}{ Estado marital } \\
\hline Sin Pareja & 18 & $(13.4)$ & 41 & $(7.3)$ \\
\hline Con Pareja & 116 & $(86.6)$ & 509 & $(92.7)$ \\
\hline \multicolumn{5}{|l|}{ Número de gestas } \\
\hline 0 & 104 & $(77.8)$ & 100 & $(18.2)$ \\
\hline $1-3$ & 30 & $(22.4)$ & 362 & $(65.8)$ \\
\hline$>3$ & 0 & & 88 & $(16.0)$ \\
\hline \multicolumn{5}{|l|}{ Tuvo abortos previos } \\
\hline $\mathrm{Si}$ & 12 & $(9.0)$ & 113 & $(20.5)$ \\
\hline \multicolumn{5}{|c|}{ Este embarazo fue planeado } \\
\hline $\mathrm{Si}$ & 67 & $(50.0)$ & 278 & $(50.5)$ \\
\hline \multicolumn{5}{|l|}{$\begin{array}{l}\text { Uso previo de método } \\
\text { anticonceptivo }\end{array}$} \\
\hline No usaba & 123 & $(91.8)$ & 461 & $(83.8)$ \\
\hline \multicolumn{5}{|c|}{ Consejería Anticonceptiva } \\
\hline $\mathrm{Si}$ & 133 & $(99.2)$ & 548 & (99.6) \\
\hline \multicolumn{5}{|c|}{ Método Evacuación uterina } \\
\hline Tratamiento Médico & 45 & $(33.6)$ & 220 & $(40.0)$ \\
\hline Tratamiento Quirúrgico & 85 & $(63.4)$ & 320 & $(58.2)$ \\
\hline AMEU & 77 & $(90.6)$ & 287 & (89.7) \\
\hline LUI & 8 & $(9.6)$ & 33 & $(10.3)$ \\
\hline Ninguno & 4 & (3.0) & 10 & $(1.8)$ \\
\hline
\end{tabular}


Cuadro 3. Factores relacionados al aborto según grupos de edad, en mujeres admitidas en el Hospital Dr. Roberto Suazo Córdova, julio 2017 a junio 2019, $\mathrm{n}=348$.

\begin{tabular}{|c|c|c|c|}
\hline \multirow{4}{*}{ Características } & \multicolumn{2}{|c|}{ Edad } & \multirow{4}{*}{ Valor $p$} \\
\hline & 10-19 años & $>19$ años & \\
\hline & $\mathrm{n}=67$ & $n=281$ & \\
\hline & $\mathrm{n}(\%)$ & $\mathrm{n}(\%)$ & \\
\hline \multicolumn{4}{|l|}{ Nivel de Escolaridad } \\
\hline Ninguno & $1(1.5)$ & $17(6.0)$ & \\
\hline Primaria & $36(53.7)$ & $161(57.3)$ & \\
\hline Secundaria & $28(41.8)$ & $87(30.9)$ & 0.183 \\
\hline Universitaria & $2(2.9)$ & $16(5.7)$ & \\
\hline \multicolumn{4}{|l|}{ Estado Civil } \\
\hline Sin Pareja & $6(8.9)$ & $23(8.2)$ & \\
\hline Con Pareja & $61(91.1)$ & $258(91.8)$ & 0.864 \\
\hline \multicolumn{4}{|l|}{ Abortos Previos } \\
\hline Con Aborto & $4(5.9)$ & $55(19.6)$ & \\
\hline Sin Aborto & 63(94.1) & $226(80.4)$ & 0.006 \\
\hline \multicolumn{4}{|l|}{ Embarazo Planeado } \\
\hline No & $31(46.3)$ & $139(49.5)$ & \\
\hline Sí & $36(53.7)$ & $142(50.5)$ & 0.154 \\
\hline \multicolumn{4}{|c|}{$\begin{array}{l}\text { Uso Método Anticonceptivo } \\
\text { Previo }\end{array}$} \\
\hline Sí & $3(4.5)$ & $48(17.1)$ & 0.007 \\
\hline No & $64(95.5)$ & $233(82.9)$ & \\
\hline \multicolumn{4}{|c|}{ Consejería Anticoncepción } \\
\hline Con Consejería & $67(100.0)$ & $281(100)$ & 0.928 \\
\hline Sin Consejería & 0 & 0 & \\
\hline \multicolumn{4}{|l|}{ Inicio Anticonceptivos } \\
\hline Sí inició Anticonceptivos & $67(50.0)$ & $281(50.1)$ & 0.82 \\
\hline No inició Anticonceptivo & $67(50.0)$ & $269(49.9)$ & \\
\hline
\end{tabular}

En relación con el inicio de un MAC después del aborto se encontró que $50.0 \%(67 / 134)$ de mujeres adolescentes y un $51.1 \%(281 / 550)$ de las mujeres mayores de 19 años eligieron iniciar un MAC. No encontrándose diferencias estadísticas significativas $(p=0.820)$ (Cuadro 3 ).

El antecedente de aborto $(p=0.006)$ y el uso previo de anticonceptivos $(p=0.007)$, estuvo asociado a que las mujeres mayores de 19 años aceptaran iniciar un anticonceptivo después del aborto (Cuadro 3). La decisión del inicio de un MAC después del procedimiento de evacuación mostró una asociación estadísticamente significativa con la evacuación operatoria tanto en las mujeres adolescentes ( $p=0.022)$ como en las mujeres mayores de 19 años $(p=0.000)$ (Cuadro 4).

En relación a la elección del MAC se encontró que entre las mujeres adolescentes que decidieron iniciar un método el principal método anticonceptivo elegido por estas, fue el acetato de medroxiprogesterona de depósito en $37.3 \%$ (25) $(p=0.782)$, seguido de los anticonceptivos combinados orales $28.4 \%(19)(p=0.171)$ y el condón $23.9 \%(16)(p=0.860)$,
Cuadro 4. Método Anticonceptivo elegido según el Método de Evacuación Uterina aplicado, Hospital Dr. Roberto Suazo Córdova. julio 2017 a junio 2019, $\mathrm{n}=670$.

\begin{tabular}{lccccc}
\hline $\begin{array}{l}\text { Edad } \\
\text { (Años) }\end{array}$ & \multicolumn{2}{c}{$\begin{array}{c}\text { Tratamiento } \\
\text { Médico } \\
\mathrm{n=265}\end{array}$} & \multicolumn{2}{c}{$\begin{array}{c}\text { Tratamiento } \\
\text { Operatorio } \\
\mathrm{n}=\mathbf{4 0 5}\end{array}$} & Valor $\boldsymbol{P}$ \\
& $\mathrm{n}$ & $(\%)$ & $\mathrm{n}$ & (\%) & \\
\hline$<19$ & & & & & \\
Inicio MAC & 17 & $(6.4)$ & 50 & $(12.3)$ & 0.022 \\
No Inicio MAC & 28 & $(10.6)$ & 35 & $(8.6)$ & \\
$>$ 19 & & & & & \\
Inicio MAC & 94 & $(35.5)$ & 185 & $(45.7)$ & 0.000 \\
No Inicio MAC & 126 & $(47.5)$ & 135 & $(33.3)$ & \\
\hline MAC-Método anticonceptivo & & & & &
\end{tabular}

$M A C=$ Método anticonceptivo

Cuadro 5. Método anticonceptivo elegido postaborto según grupo de edad. Hospital Dr. Roberto Suazo Córdova. julio 2017 a junio 2019, n=348.

\begin{tabular}{|c|c|c|c|c|c|}
\hline \multirow{3}{*}{$\begin{array}{l}\text { Métodos } \\
\text { anticonceptivos }\end{array}$} & \multicolumn{4}{|c|}{ Edad } & \multirow{3}{*}{ Valor $p$} \\
\hline & \multicolumn{2}{|c|}{$\begin{array}{c}10-19 \text { años } \\
(n=67)\end{array}$} & \multicolumn{2}{|c|}{$\begin{array}{l}>19 \text { años } \\
(n=281)\end{array}$} & \\
\hline & $\mathrm{n}$ & $(\%)$ & $\mathrm{n}$ & $(\%)$ & \\
\hline $\begin{array}{l}\text { Acetato de } \\
\text { Medroxiprogesterona }\end{array}$ & 25 & $(37.3)$ & 110 & (39.1) & 0.782 \\
\hline Combinados Orales & 19 & (28.4) & 58 & (20.6) & 0.171 \\
\hline Condón & 16 & $(23.9)$ & 70 & $(24.9)$ & 0.860 \\
\hline Dispositivo Intrauterino & 4 & $(6.0)$ & 22 & $(7.8)$ & 0.603 \\
\hline Implantes & 1 & $(1.5)$ & 1 & $(0.4)$ & 0.696 \\
\hline Otro hormonal & 1 & $(1.5)$ & 4 & $(1.4)$ & 0.999 \\
\hline Otros de barrera & 1 & $(1.5)$ & 3 & (1.1) & 0.999 \\
\hline Ligadura tubárica & 0 & $(0.0)$ & 11 & (3.9) & - \\
\hline Abstinencia periódica & 0 & $(0.0)$ & 2 & $(0.7)$ & - \\
\hline
\end{tabular}

esta distribución se mantuvo en las mujeres mayores de 19 años con el acetato de medroxiprogesterona elegido en $39.1 \%$ (110), anticonceptivos combinados $20.6 \%$ (58) y condón $24.9 \%$ (70); no encontrándose diferencias estadísticamente significativas (Cuadro 5).

La elección de un método LARC, fue únicamente de 7.4\% (5) entre las adolescentes y de $8.2 \%$ (23) entre las mujeres mayores de 19 años (Cuadro 5).

\section{DISCUSIÓN}

Los resultados mostraron que la edad no fue un factor determinante para la decisión del inicio de un método anticonceptivo después de un aborto. Ambos grupos, adolescentes y adultas se comportaron de igual manera en relación con la elección de un método anticonceptivo en el período de postaborto, independientemente de haber recibido consejería anticonceptiva. 
El 91.8\% (123) de las adolescentes no usaba anticonceptivos antes de este aborto. Además, se encontró que el $50.0 \%$ (67) de los embarazos fueron no planeados, lo que está en concordancia con estudios que reportan que a nivel de Latinoamérica y el Caribe las tasas de embarazos no planeados son altas y fundamentalmente relacionadas con la falta de uso de métodos anticonceptivos siendo las adolescentes uno de los grupos más vulnerables. ${ }^{1}$

En el grupo de las adolescentes se encontró que el $44.8 \%$ (60) tenía nivel de escolaridad secundaria o universitaria. Algunos estudios reportan el nivel de escolaridad como un factor protector contra los embarazos repetidos. ${ }^{5}$

Los métodos LARC solo se eligieron en un $7.4 \%$ (5) en este grupo de mujeres adolescentes, con el riesgo de que no haya un apego adecuado al método elegido. No hubo diferencias estadísticamente significativas con el grupo de mujeres mayores de 19 años en las que la elección de un Método LARC fue solamente de $8.2 \%$ (23). Este resultado confirma lo que se ha publicado en otros estudios como el realizado en Luanda, Angola que reportó que solo el $2.7 \%$ de las mujeres usaron un método LARC después de un aborto. ${ }^{11}$

El hecho de que solo el $7.4 \%$ de mujeres adolescentes y $8.2 \%$ de las mujeres adultas eligieron usar LARC a pesar de haber sufrido un aborto y de que el embarazo en muchos casos no fue planeado indica que las adolescentes y adultas en nuestro medio son usuarias erráticas de MAC. En el caso específico del seguimiento que estas adolescentes puedan tener después de un aborto es importante para la prevención de embarazos no planeados ya que gran parte de ellas terminan abandonando el contacto con el servicio de salud, principalmente entre aquellas con un nivel de escolaridad menor. ${ }^{5}$

La forma de evacuación uterina puede influir sobre el tipo de anticonceptivo que la mujer elija después de un aborto. A pesar de tener opciones anticonceptivas similares, el tratamiento médico del aborto representa un reto único para proveer un amplio rango de métodos anticonceptivos sobre todo los métodos LARC ya que mientras todos los métodos pueden aplicarse seguramente el mismo día del tratamiento operatorio, el manejo con tratamiento médico requiere una segunda visita sobre todo cuando se trata de los métodos LARC. ${ }^{6}$ Esto está en concordancia con los resultados encontrados en este estudio en el que se observó que tanto en las adolescentes como las mujeres mayores de 19 años la elección de un método anticonceptivo se asoció a la evacuación por método operatorio.

Un estudio de Morris y colaboradores realizado en Luanda, Angola en el 2018, reportó que las mujeres con historia de abortos tienen más probabilidades de aceptar el inicio de un anticonceptivo moderno en comparación con aquellas que nunca han tenido un aborto. ${ }^{11}$ Este resultado está en concordancia con los resultados de este estudio que muestran que las mujeres mayores aceptan más el uso de anticonceptivos después de un aborto, pero esto no tuvo el mismo resultado en las adolescentes.

La decisión de elegir un método LARC fue muy baja tanto en las adolescentes como en las adultas; ambos grupos optaron más por escoger Acetato de Medroxiprogesterona, 37.3\% las adolescentes y $39.1 \%$ las mayores de 19 años, seguido de los anticonceptivos orales, $28.6 \%$ las adolescentes y $20.6 \%$ las mujeres mayores y el condón, $23.9 \%$ las adolescentes y $24.9 \%$ las mayores. La elección baja de un método LARC fue similar a lo reportado por Bustillo y González en el estudio realizado en cuatro hospitales de Honduras, enero 2017-julio 2019. En dicho estudio se indicó que se debe brindar información y consejería apropiada para la elección de LARC, el cual a su vez debe estar en todas las instituciones de salud, sin costo y de libre acceso. ${ }^{12}$ También, el estudio de Morris y colaboradores en Angola en el 2018, se encontró que el uso de métodos LARC fue bajo y que el método más aceptado fue el condón. ${ }^{11}$ Esto es importante de tener en consideración porque se ha descrito que la mayoría de las adolescentes deciden cambiar por un método anticonceptivo más seguro después de un aborto. ${ }^{13}$ Sin embargo, esto no se observó en nuestro estudio ya que la aceptación de métodos LARC fue baja.

En un estudio de Omideyi, AK y colaboradores en Southwest, Nigeria en el 2011, describió que son factores con gran influencia en la aceptación de anticoncepción, el nivel de escolaridad, estado socioeconómico, inmadurez, intervalo intergenésico corto y estigma social. ${ }^{14}$ En este estudio no se consideraron varias de estas variables.

Los cuidados postaborto son una herramienta de gran importancia para todas las mujeres y particularmente para adolescentes ya que permite incrementar el acceso a una amplia gama de MAC, principalmente en nuestro medio donde los métodos LARC tienen menor aceptación como confirmamos en este estudio. Por otro lado, a pesar de que las mujeres recibieron consejería en planificación familiar, no hubo mayor impacto sobre la decisión de iniciar MAC y principalmente aceptar un método LARC.

Lo antes mencionado indica que la aceptación de un método LARC es un reto en las adolescentes, esto es debido a que las características ambientales y de desarrollo influyen sobre su comportamiento anticonceptivo. La elección de un método reversible de acción corta a uno de larga acción está influenciada por edad, estado civil, paternidad o fuentes de información en anticoncepción; por lo que el acceso a anticoncepción, educación de calidad y asesoramiento adecuado deben convertirse en una prioridad en la atención en planificación familiar. ${ }^{8,15-17}$

La seguridad y la eficacia del método anticonceptivo elegido después de un aborto son factores importantes a tener en cuenta ya que se sabe que los métodos LARC tanto el DIU como el Implante son seguros y eficaces para prevenir los embarazos no planeados o los abortos a repetición y debe de considerarse que no requieren adherencia por parte de las pacientes de allí que en adolescentes y mujeres jóvenes los métodos LARC son altamente beneficiosos y efectivos ya que estas mujeres son más propensas a la falta de cumplimiento de los métodos que requieren adherencia. ${ }^{18}$ Sin embargo, aun considerando todo lo anterior en un estudio de Evens E. y colaboradores en Kenia en el 2014, reportaron que menos adolescentes recibieron un MAC en comparación con mujeres adultas, lo cual puede deberse a temor a la infertilidad, efectos secundarios o falta de conocimiento. ${ }^{19}$ Los hallazgos de este estudio muestran que sólo la 
mitad de las adolescentes eligieron iniciar un MAC; esto a pesar de que todas las adolescentes recibieron una consejería, nos hace considerar de que ésta no tuvo la calidad necesaria para influir sobre la decisión de aceptar un método anticonceptivo. Por otro lado, se debe tomar en cuenta el aspecto cultural de las adolescentes y su entorno que se convierte en una limitante para que estas puedan aceptar un método. El impacto en la salud pública, en términos de costos y beneficios de satisfacer las necesidades anticonceptivas de los adolescentes es claro; por lo que satisfacer las necesidades insatisfechas de anticoncepción moderna de adolescentes reduciría los embarazos no planeados. ${ }^{20}$ Deben procurarse prácticas que permitan cuidar de estas adolescentes de una manera más adecuada tomando en consideración su edad, bases culturales y perfiles de riesgo. ${ }^{21,22}$

Entre las limitantes encontradas, se puede mencionar que al ser un estudio retrospectivo sobre la base del SIP que está constituido por variables preestablecidas, no se pudo tener acceso a otros datos que pudieran influir sobre el uso de métodos anticonceptivos al momento de la atención tal como accesibilidad y disponibilidad de los métodos anticonceptivos.

En conclusión, el inicio de un MAC postaborto no estuvo asociado a la edad. En el grupo de mujeres mayores de 19 años se asoció al antecedente de abortos y el uso previo de algún método anticonceptivo. La elección de un MAC estuvo asociado al método de evacuación ya sea AMEU o LUI, y el principal método anticonceptivo elegido fue el Acetato de Medroxiprogesterona con una baja aceptación de métodos LARC. Se requiere evaluar y mejorar el abordaje a la información sobre los méto- dos anticonceptivos mejorando el plan de consejería, promover y garantizar el acceso y la disponibilidad de los métodos anticonceptivos sobre todo los métodos LARC para asegurar un mayor apego al método que la mujer elija.

\section{CONTRIBUCIONES}

Todos los autores desarrollaron la idea del estudio, revisaron base de datos, hicieron el control de calidad. RCB coordinó la escritura del documento, elaboró los cuadros, incorporó recomendaciones. Todos los autores revisaron y aprobaron la versión final del documento.

\section{AGRADECIMIENTOS}

Se agradece a la OMS/OPS y CLAP/SMR. Al Dr. Lester David Castro Paz, Maestría en Ciencia de la Salud, en el área Materna y Perinatal, en la Universidad de Campinas, Brasil por su apoyo en la estructuración del documento final.

\section{DESCARGO DE RESPONSABILIDAD}

Las opiniones expresadas en este artículo, realizado con el apoyo financiero de la OMS y de la OPS, son de exclusiva responsabilidad de sus autores y no representan necesariamente la opinión de ambas instituciones.

\section{DETALLES DE AUTOR(ES)}

Rigoberto Castro, Médico Gineco-Obstetra; rcastrob561@ hotmail.com

Jamilette Paz, Médica Gineco-Obstetra; jamipaz@gmail.com

\section{REFERENCIAS}

1. Bahamondes L, Villarroel C, Frias Guzman N, Oizerovich S, VelazquezRamirez N, Monteiro I. The use of long-acting reversible contraceptives in Latin America and the Caribbean: current landscape and recommendations. Hum Reprod Open. 2018;2018(1): hox030. [citado 19 de septiembre 2019]. Disponible en http://dx.doi.org/10.1093/hropen/hox030

2. Rashid S, Moore JE, Timmings C, Vogel JP, Ganatra B, Khan DN, et al. Evaluating implementation of the World Health Organization's Strategic Approach to strengthening sexual and reproductive health policies and programs to address unintended pregnancy and unsafe abortion. Reprod Health. 2017;14(1):153. [citado19septiembre2019]. Disponible en http:// dx.doi.org/10.1093/hropen/hox030

3. Chinchilla AL, Castellanos k. Caracterización del aborto en Hospitales Públicos de Honduras. 2010-2011. Tegucigalpa: Secretaría de Salud, OPSFIGO; 2011

4. Margherio C. Centering female agency while investigating contraceptive use: a case study in Agincourt, South Africa. Int J Equity Health. 2019;18(1):60. [citado19septiembre2019]. Disponible en http://dx.doi. org/10.3109/13625181003782852

5. Govender D, Naidoo S, Taylor M. Prevalence and risk factors of repeat pregnancy among South African Adolescent Females. Afr J Reprod Health. 2019;23(1):73-87. [citado 19 septiembre 2019]. Disponible en http://dx.doi. org/10.29063/ajrh2019/v23i1.

6. Rocca CH, Goodman S, Grossman D, Cadwallader K, Thompson KMJ, Talmont E, et al. Contraception after medication abortion in the United States: results from a cluster randomized trial. Am J Obstet Gynecol. 2018;218(1):107e1-e8. [ citado 19 septiembre 2019]. Disponible en http:// dx.doi.org/10.1016/j.ajog.2017.09.

7. Tayo A, Akinola O. contraceptive knowledge and usage amongst female secondary school students in Lagos, southwest Nigeria. J Pub Health. 2011;3(1):34-7. [citado 19 febrero 2021]. Disponible en http://www.academicjournals.org/jphe
8. Sedlecky K, Stanković Z. Contraception for adolescents after abortion. Eur J Contracept Reprod Health Care. 2016;21(1):4-14. [Citado 9 febrero 2021]. Disponible en https://doi.org/10.3109/13625187.2015.1082175

9. Madden T, Secura GM, Allsworth JE, Peipert JF. Comparison of contraceptive method chosen by women with and without a recent history of induced abortion. Contraception. 2011;84(6):571-7. [Citado 9 febrero 2021]. Disponible en http://dx.doi.org/10.1016/j.contraception.2011.03.018

10. Gómez Ponce de León A. Líneas estratégicas para la Red MUSA (Asistencia a Mujeres en Situación de Aborto) en América Latina y Caribe [Internet]. Washington D.C.: Organización Panamericana de la Salud; 2015. [citado14 febrero 2021]. Disponible en: https://bit.ly/3gUY2yn

11. Morris N, Prata N. Abortion history and its association with current use of modern contraceptive methods in Luanda, Angola. Open Access J Contracept. 2018; 9:45-55. [Citado 10 febrero 2021] Disponible en https://doi. org/10.2147/OAJC. $\$ 164736$

12. Bustillo $\mathrm{C}$, González $\mathrm{G}$. Uso de métodos anticonceptivos de larga acción en mujeres adolescentes en hospitales de Honduras. REV MED HONDUR.2020; 88(2): 99-103. [citado el 16 de julio del 2021]. Disponible en: http://www.bvs.hn/RMH/pdf/2020/html/Vol88-2-2020.htm

13. Moreau C, Trussell J, Desfreres J, Bajos N. Peri-abortion contraceptive use in the French islands of Guadeloupe and La Réunion: variation in the management of post-abortion care. Eur J Contracept Reprod Health Care. 2010;15(3):186-96. [citado 9 febrero 2021]. Disponible en http://dx.doi. org/10.3109/13625181003782852

14. Omideyi AK, Akinyemi Al, Aina OI, Adeyemi AB, Fadeyibi OA, Bamiwuye SO, et al. Contraceptive practice, unwanted pregnancies and induced abortion in Southwest Nigeria. Glob Public Health. 2011;6 (Suppl 1): S5272. [ Citado 9 febrero 2021]. Disponible en http://dx.doi.org/10.1080/17441 692.2011.594073

15. Makenzius M, Faxelid E, Gemzell-Danielsson K, Odero TMA, KlingbergAllvin M, Oguttu M. Contraceptive uptake in post abortion Care-Secondary 
outcomes from a randomised controlled trial, Kisumu, Kenya. PLoS One. 2018;13(8): e0201214. [Citado 9 febrero 2021]. Disponible en http://dx.doi. org/10.1371/journal.pone.0201214

16. Moreau C, Trussell J, Bajos N. Contraceptive paths of adolescent women undergoing an abortion in France. J Adolesc Health. 2012;50(4):389-94. [Citado 9 febrero 2021]. Disponible en http://dx.doi.org/10.1016/j.jadohealth.2011.07.013

17. Zgliczynska M, Szymusik I, Sierocinska A, Bajaka A, Rowniak M, Sochacki-Wojcicka N, et al. Contraceptive behaviors in polish women aged 18-35a cross-sectional study. Int J Environ Res Public Health. 2019;16(15):2723. [ Citado 9 febrero 2021]. Disponible en https://doi.org/10.3390/ijerph16152723

18. Gemzell-Danielsson K, Kopp HK. Post abortion contraception. Womens Health (Lond). 2015;11(6):779-84. [Citado 10 febrero 2021]. Disponible en https://journals.sagepub.com/doi/pdf/10.2217/whe.15.72

19. Evens E, Otieno-Masaba R, Eichleay M, McCarraher D, Hainsworth G, Lane $C$, et al. Post-abortion care services for youth and adult clients in Kenya: a comparison of services, client satisfaction and provider attitudes. J Biosoc Sci. 2014;46(1):1-15. [citado 9 febrero 2021]. Disponible en http:// dx.doi.org/10.1017/s0021932013000230

20. Darroch JE, Woog V, Bankole A, Ashford LS. Costs and benefits of meeting the contraceptive needs of adolescents [Internet]. New York: Guttmacher Institute; 2016. [citado 19 septiembre 2019]. Disponible en: https://www. guttmacher.org/report/adding-it-meeting-contraceptive-needs-of-adolescents

21. Cordova Pozo K, Chandra-Mouli V, Decat P, Nelson E, De Meyer S, Jaruseviciene $L$, et al. Improving adolescent sexual and reproductive health in Latin America: reflections from an International Congress. Reprod Health. 2015; 12:11. [ Citado 9 febrero 2021]. Disponible en http://dx.doi. org/10.1186/1742-4755-12-11

22. Fleming N, O'Driscoll T, Becker G, Spitzer RF, Leming N, O'Driscoll $T$, Becker G, Spitzer RF; CANPAGO COMMITTEE. Adolescent Pregnancy Guidelines. J Obstet Gynaecol Can. 2015 Aug;37(8):740-756. Adolescent Pregnancy Guidelines. J Obstet Gynaecol Can. 2015;37(8):740-56. [Citado 9 febrero 2021]. Disponible en http://dx.doi.org/10.1016/s17012163(15)30180-8.
ABSTRACT. Background: The rate of unintended pregnancies in Latin America is among the highest in the world. Adolescents are among the most vulnerable groups for unintended pregnancies with percentages of $32 \%$ to $45 \%$. Objective: To describe the factors associated with the choice of a contraceptive by women after an abortion at the Dr. Roberto Suazo Córdova Hospital, La Paz, Honduras, from July 2017 to June 2019. Method: Retrospective descriptive study, the data were obtained using the Perinatal Informatic Sistem (SIP Abortion) in the SIP 4.16. Results: $50.0 \%$ $(67 / 134)$ of the adolescents and $50.1 \%(281 / 550)$ of those over 19 years of age chose a postabortion contraceptive. The start of the contraceptive was related to a history of abortion $(p=0.006)$ and previous use of contraceptives $(p=0.007)$. The most popular contraceptives were: Medroxyprogesterone acetate, combined oral contraceptives and condoms. The choice of contraceptive was related to instrumental evacuation of abortion $(p=0.022)$ in adolescents and $(p=0.000)$ in those over 19 years of age. Long-acting reversible methods were less chosen. Discussion: Postabortion contraceptive choice was low. The start of contraceptives was related to a history of abortion, previous use of contraceptives and instrumental evacuation. The most popular contraceptives were short-acting temporary contraceptives, and the least chosen, longacting temporary contraceptives. Access to information must be strengthened, counseling improved, access and availability of contraceptives, especially long-acting contraceptives, guaranteed to ensure adherence to the contraceptive method.

Keywords: Abortion, Adolescent, Counseling, Contraceptives. 